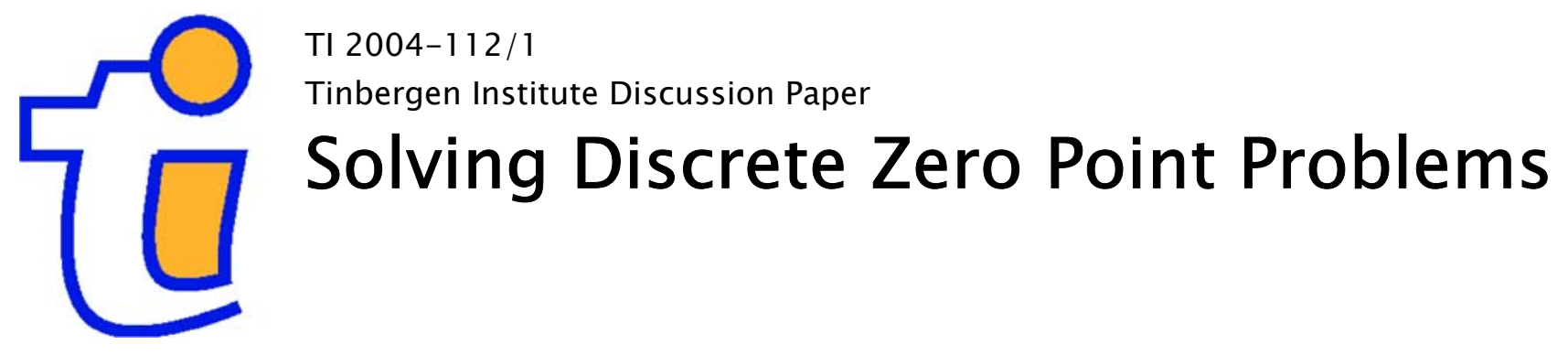

Gerard van der Laan'

Dolf Talman²

Zaifu Yang ${ }^{3}$

${ }^{\prime}$ Faculty of Economics and Business Administration, Vrije Universiteit Amsterdam, and Tinbergen Institute,

2 Department of Econometrics \& Operations Research, and CentER, Tilburg University,

${ }^{3}$ Faculty of Business Administration, Yokohama National University, Japan. 
Tinbergen Institute

The Tinbergen Institute is the institute for economic research of the Erasmus Universiteit Rotterdam, Universiteit van Amsterdam, and Vrije Universiteit Amsterdam.

Tinbergen Institute Amsterdam

Roetersstraat 31

1018 WB Amsterdam

The Netherlands

Tel.: $\quad+31(0) 205513500$

Fax: $\quad+31(0) 205513555$

Tinbergen Institute Rotterdam

Burg. Oudlaan 50

3062 PA Amsterdam

The Netherlands

Tel.: $\quad+31(0) 104088900$

Fax: $\quad+31(0) 104089031$

Please send questions and/or remarks of nonscientific nature to driessen@tinbergen.nl.

Most TI discussion papers can be downloaded at http://www.tinbergen.nl. 


\title{
Solving Discrete Zero Point Problems ${ }^{1}$
}

\author{
Gerard van der Laan, ${ }^{2}$ Dolf Talman, ${ }^{3}$ Zaifu Yang ${ }^{4}$
}

October 15, 2004

${ }^{1}$ This research was carried out while Gerard van der Laan and Zaifu Yang were visiting the CentER for Economic Research, Tilburg University. The visit of Zaifu Yang has been made possible by financial support of CentER and the Netherlands Organization for Scientific Research (NWO).

${ }^{2}$ G. van der Laan, Department of Econometrics and Tinbergen Institute, Vrije Universiteit, De Boelelaan 1105, 1081 HV Amsterdam, The Netherlands, glaan@econ.vu.nl

${ }^{3}$ A.J.J. Talman, Department of Econometrics \& Operations Research and CentER, Tilburg University, P.O. Box 90153, 5000 LE Tilburg, The Netherlands, talman@uvt.nl

${ }^{4}$ Z. Yang, Faculty of Business Administration, Yokohama National University, Yokohama 2408501, Japan, zyang@business.ynu.ac.jp 


\begin{abstract}
In this paper an algorithm is proposed to find a discrete zero point of a function on the collection of integral points in the $n$-dimensional Euclidean space $\mathbb{R}^{n}$. Starting with a given integral point, the algorithm generates a finite sequence of adjacent integral simplices of varying dimension and terminates with, under certain convergency conditions, a vertex, which yields a discrete zero point of the function under consideration.
\end{abstract}

Keywords: Discrete zero point, discrete fixed point, simplicial algorithm, triangulation.

AMS subject classifications: 47H10, 54H25, 55M20, 90C33, 91B50. 


\section{Introduction}

We consider the problem of finding an integral point $x^{*} \in \mathbb{Z}^{n}$ such that

$$
f\left(x^{*}\right)=0^{n}
$$

where $0^{n}$ is the $n$-vector of zeros, $Z^{n}$ is the set of vectors in the $n$-dimensional Euclidean space $\mathbb{R}^{n}$ with all components being integer, and $f$ is a function from $\mathbb{Z}^{n}$ to $\mathbb{R}^{n}$. Such an integral point $x^{*}$ is called a discrete zero point of $f$. Recently, the existence problem of a discrete zero point (or equivalently fixed point of the function $g(x)=f(x)+x$ ) has been independently investigated by Iimura, Murota and Tamura (2004), Danilov and Koshevoy (2004) and Yang (2004a,b). These papers were all inspired by Iimura's (2003) incorrect discrete fixed point theorem. In Iimura et al. (2004) a corrected version of Iimura's discrete fixed point theorem has been established, while a similar theorem is given by Danilov and Koshevoy (2004). In both papers their theorems concern functions that exhibit the socalled direction preserving property, which can be seen as the counterpart of the continuity property for functions defined on the Euclidean space $\mathbb{R}^{n}$. This class of direction preserving functions on $Z^{n}$ is introduced by Iimura (2003). In Yang (2004a,b) several more general discrete fixed (or zero) point theorems have been established, which contain the results of Iimura et al. (2004) and Danilov and Koshevoy (2004) as special cases. The existence results of Yang hold for the class of so-called locally gross direction preserving mappings, which is substantially more general and richer than the class of Iimura's direction preserving mappings. Besides establishing these more general existence results, Yang (2004a) also initiated the study of discrete nonlinear complementarity problems and provided several general theorems for the existence of solutions for this class of problems. All this literature, however, is not concerned with the problem of actually finding an integral solution. In fact, all existence proofs are nonconstructive ones.

The main aim of this paper is to provide a finite and efficient algorithm for finding a discrete zero point of direction preserving functions. Much of the recent literature on the approximation of fixed or zero points has its root in the pioneering work of Scarf (1967, 1973). To find an approximate equilibrium price vector in economic general equilibrium models, Scarf introduced a procedure that was guaranteed to converge, based on the nowadays well-known Lemke-Howson argument. Subsequent algorithms, developed by Eaves (1972), Merrill (1972), van der Laan and Talman (1979) among others, substantially refined Scarf's original algorithm in order to accelerate its speed of convergence and to extend its applicability. As a consequence of these types of algorithms, by a limit argument, fixed point theorems like those of Brouwer and Kakutani can be proved in a constructive way. For comprehensive treatments on fixed point algorithms developed at various stages, we refer to Allgower and Georg (1990), Todd (1976) and Yang (1999). 
In this paper we will adapt the so-called $2 n$-ray algorithm, introduced by van der Laan and Talman (1981), to find a discrete zero point of a direction preserving function $f$ : $\mathbb{Z}^{n} \rightarrow \mathbb{R}^{n}$. Recall that for the existence of a zero point of a function on the $n$-dimensional Euclidean space $\mathbb{R}^{n}$ two types of conditions are needed, namely some kind of continuity and a boundary condition, making it possible to restrict the domain of the function on which a zero point exists to a convex and compact set in $\mathbb{R}^{n}$. Of course, similar boundary conditions are needed for the discrete case. In this paper we will provide two of such boundary conditions ensuring the existence of a discrete zero point of a direction preserving function. The first one is similar to a condition stated in Merrill (1972) for the continuous case on $\mathbb{R}^{n}$. The second one has been stated already in Yang (2004b) and can be seen as the counterpart for discrete functions of the famous Borsuk-Ulam condition for the existence of a zero point of a continous function. For each one of these boundary conditions, we show that a modification of the 2n-ray algorithm will find a zero point within a finite number of steps for any direction preserving function satisfying this boundary condition. Thus, by this approach we give a constructive and combinatorial proof for a new discrete zero point theorem for functions satisfying a Merrill type of boundary condition and furthermore we establish a constructive and combinatorial proof for a discrete zero point theorem of Yang (2004b) for functions satisfying a Borsuk-Ulam type of boundary condition.

The algorithm providing these constructive proofs is based on a triangulation (or socalled simplicial subdivision) of $\mathbb{R}^{n}$, constructed in such a way that the set of vertices of the triangulation is equal to $\mathbb{Z}^{n}$ and the mesh size of each simplex in the triangulation is equal to one according to the maximum norm. Then, starting with a given integral point in $\mathbb{Z}^{n}$, the algorithm generates a finite sequence of adjacent simplices of varying dimension and terminates with a simplex satisfying that one of its vertices is a discrete zero point. It is worth pointing out that all previous works on the computation of fixed (or zero) points typically find an approximate solution for a continuous function on $\mathbb{R}^{n}$. In sharp contrast, when applying to the discrete case, the 2n-ray algorithm always finds an exact solution within a finite number of steps. The algorithm we apply in this paper is a so-called integerlabeling algorithm, meaning that we label each vertex of the triangulation with an integer value determined by the function value of the vertex.

The remaining of this paper is organized as follows. In Section 2 we present some basic concepts and the two boundary conditions mentioned above. In Sections 3 and 4 we establish the discrete zero point theorems under these two boundary conditions, respectively, and we describe the algorithms to provide the constructive and combinatorial proofs of both theorems. Finally, concluding remarks are given in Section 5. 


\section{Basic concepts and conditions}

We first give some general notation. For given positive integer $n$, let $N$ denote the set $\{1,2, \ldots, n\}$. For $x, y \in \mathbb{R}^{n}, x \cdot y$ stands for the inner product of $x$ and $y$. For $i \in N, e(i)$ denotes the $i$ th unit vector of $\mathbb{R}^{n}$ and $e(-i)=-e(i)$. Given a set $D \subset \mathbb{R}^{n}, \operatorname{Co}(D)$ and $\operatorname{Bd}(D)$ denote the convex hull of $D$ and the (relative) boundary of $D$, respectively.

For any integer $t, 0 \leq t \leq n$, the convex hull of $t+1$ affinely independent points $x^{1}, \ldots, x^{t+1}$ in $\mathbb{R}^{n}$ is called a $t$-dimensional simplex or simply a $t$-simplex or simplex and will be denoted by $\sigma$ or $\sigma\left(x^{1}, \ldots, x^{t+1}\right)$. The extreme points $x^{1}, \ldots, x^{t+1}$ of a $t$-simplex $\sigma\left(x^{1}, \ldots, x^{t+1}\right)$ are called the vertices of $\sigma$. The convex hull of any subset of $k$ vertices of a $t$-simplex $\sigma, 1 \leq k \leq t-1$, is called a face or $k$-face of $\sigma$. A $k$-face of a $t$-simplex $\sigma$ is called a facet of $\sigma$ if $k=t-1$, i.e., if the number of vertices is just one less than the number of vertices of the simplex. A simplex is said to be integral if all of its vertices are integral vectors, i.e., all vertices are points in $Z^{n}$.

Given an $m$-dimensional convex set $D$, a collection $\mathcal{T}$ of $m$-dimensional simplices is a triangulation or simplicial subdivision of the set $D$, if (i) $D$ is the union of all simplices in $\mathcal{T}$, (ii) the intersection of any two simplices of $\mathcal{T}$ is either empty or a common face of both, and (iii) any neighborhood of any point in $D$ only meets a finite number of simplices of $\mathcal{T}$. In this paper we use the $K^{\prime}$-triangulation of $\mathbb{R}^{n}$ with grid size equal to 1 , proposed by Todd (1978). This simplicial subdivisions is such that all its simplices are integral. For some given integral vector $w \in \mathbb{Z}^{n}$, the $K^{\prime}$-triangulation originating in $w$, denoted by $K^{\prime}(w)$, is the collection of all $n$-dimensional simplices $\sigma\left(x^{1}, \ldots, x^{n+1}\right)$ such that

(i) $x^{1} \in \mathbb{Z}^{n}$ and

(ii) $x^{i+1}=x^{i}+s_{\pi(i)} e(\pi(i)), i=1, \ldots, n$, where $\pi=(\pi(1), \ldots, \pi(n))$ is a permutation of the elements in $N$ and where $s \in\{-1,1\}^{n}$ is chosen such that $s_{j}=-1$ if $x_{j}^{1}<w_{j}$ and $s_{j}=1$ if $x_{j}^{1}>w_{j}$.

So, for a simplex $\sigma\left(x^{1}, \ldots, x^{n+1}\right)$ in $K^{\prime}(w)$ it holds that each vertex $x^{i+1}, i \geq 1$, is obtained from its predecessor $x^{i}$ by adding or substracting a unit vector, where for $j \in N$ it is required that $e(j)$ is added (substracted) if $x_{j}^{1}$ is strictly greater (smaller) than $w_{j}$ (so, when $x_{j}^{1}=w_{j}$ both possibilities are allowed). For a more detailed description we refer to Todd (1978).

Two integral points $x$ and $y$ in $\mathbb{Z}^{n}$ are said to be cell connected if $\max _{h \in N}\left|x_{h}-y_{h}\right| \leq 1$, i.e., their distance is less than or equal to one according to the maximum norm. In other words, two integral points $x$ and $y$ are cell connected if and only if both $x$ and $y$ belong to the set $\{0,1\}^{n}+\{q\}$ for some $q \in \mathbb{Z}^{n}$. Observe that any two vertices of a simplex of the $K^{\prime}(w)$-triangulation are cell connected. We now recall the following definition.

Definition 2.1 A function $f: \mathbb{Z}^{n} \rightarrow \mathbb{R}^{n}$ is direction preserving if for any two cell 
connected points $x$ and $y$ in $\mathbb{Z}^{n}$ it holds that

$$
f_{h}(x) f_{h}(y) \geq 0 \text { for all } h \in N \text {. }
$$

The class of direction preserving functions is due to Iimura (2003). Observe that the property of direction preserving prevents coordinatewisely that the function jumps from a positive value to a negative value within one cell. In this sense it replaces continuity for functions on $\mathbb{R}^{n}$.

Finally, we state two boundary conditions under which a discrete zero point exists.

Definition 2.2 (1) A function $f: \mathbb{Z}^{n} \rightarrow \mathbb{R}^{n}$ satisfies the negativity boundary condition if there exist vectors $m, v, M \in \mathbb{Z}^{n}$ with $m_{h}+1<v_{h}<M_{h}-1$ for every $h \in N$, such that for every integral vector $x$ on the boundary of the set $C^{n}=\left\{y \in \mathbb{R}^{n} \mid m \leq y \leq M\right\}$ it holds that

$$
(x-v) \cdot f(x)<0 .
$$

(2) A function $f: \mathbb{Z}^{n} \rightarrow \mathbb{R}^{n}$ satisfies the antipodal boundary condition if there exists $u \in \mathbb{Z}^{n}$ with $u_{h}>1$ for all $h \in N$, such that for any two cell connected integral points $x$ and $y$ (including the case that $x=y$ ) on the boundary of the set $U^{n}=\left\{x \in \mathbb{R}^{n} \mid-u \leq x \leq u\right\}$ it holds that

$$
f_{h}(x) f_{h}(-y) \leq 0, \quad h=1, \ldots, n .
$$

The first condition can be seen as a discrete analogue of a condition for a continuous function due to Merrill (1972). The second one can be viewed as a discrete analogue of a weak version of the Borsuk-Ulam antipodal condition for a continuous function saying that $f_{h}(x) f_{h}(-x) \leq 0$ for all $h$ when $x$ is on the boundary of $U^{n}$. It can be shown that under this condition a continuous function has a zero point; see for instance van der Laan (1984).

\section{Existence of discrete zero point under the negativ- ity boundary condition}

In this section we present a new discrete zero point theorem for functions satisfying the negativity boundary condition and provide a constructive and combinatorail proof for the theorem.

Theorem 3.1 Let $f: \mathbb{Z}^{n} \rightarrow \mathbb{R}^{n}$ be a direction preserving function satisfying the negativity boundary condition for some $m, v, M \in \mathbb{Z}^{n}$. Then $f$ has a discrete zero point $x^{*} \in \mathbb{Z}^{n}$, i.e., $f\left(x^{*}\right)=0^{n}$. 
To prove the theorem, we adapt the 2n-ray algorithm of van der Laan and Talman (1981), which was originally introduced to approximate a fixed point of a continuous function, to the current discrete setting. To do so, we first introduce an integer labeling rule, assigning to each integral point an integer label based on the function value.

Definition 3.2 For given $f: \mathbb{Z}^{n} \rightarrow \mathbb{R}^{n}$, the $(2 n+1)$-labeling rule $l: \mathbb{Z}^{n} \rightarrow(-N) \cup N \cup$ $\{0\}$ is specified as follows. For any given $x \in \mathbb{Z}^{n}$, set $l(x)=0$ if $f(x)=0^{n}$. Otherwise, let $k$ be the first nonzero component of $f(x)$ and set $l(x)=k$ if $f_{k}(x)>0$ and $l(x)=-k$ if $f_{k}(x)<0$.

The algorithm is applied on $K^{\prime}(v)$, i.e., on the $K^{\prime}$-triangulation with respect to the integral point $v$ as specified in Theorem 3.1. Let $\mathcal{I}$ be the family of subsets $S$ of $(-N) \cup N$ such that $k \in S$ implies $-k \notin S$. For $T \in \mathcal{I}$ define the set $A(T)$ by

$$
\begin{gathered}
A(T)=\left\{x \in \mathbb{R}^{n} \mid \quad x_{i} \geq v_{i} \text { if } i \in T ; x_{i} \leq v_{i} \text { if }-i \in T\right. \\
\text { and } \left.x_{i}=v_{i} \text { otherwise }\right\} .
\end{gathered}
$$

The $K^{\prime}(v)$-triangulation of $\mathbb{R}^{n}$ induces a triangulation of $A(T)$ for every $T \in \mathcal{I}$. More precisely, the set $A(T)$ is subdivided into $t$-simplices $\sigma\left(x^{1}, \ldots, x^{t+1}\right)$, where $t=|T|, x^{1} \in$ $A(T) \cap \mathbb{Z}^{n}$ and $x^{i+1}=x^{i}+e(\pi(i)), i=1, \ldots, t$, for some permutation $\pi=(\pi(1), \ldots, \pi(t))$ of the elements of $T$. Any facet $\tau$ of a $t$-simplex $\sigma$ in $A(T)$ is either a facet of exactly one other $t$-simplex $\sigma^{\prime}$ in $A(T)$ or lies on the boundary of $A(T)$ and is a $(t-1)$-simplex in $A(T \backslash\{k\})$ for some uniquely determined $k \in T$. Moreover, all simplices in $A(T)$ are integral and any two vertices of a simplex in $A(T)$ are cell connected. Given a $t$-simplex $\sigma$ in $A(T)$, a facet $\tau$ of $\sigma$ is said to be T-complete if

$\{l(x) \mid x$ is a vertex of $\tau\}=T$.

In other words, the $t$ vertices of $\tau$ are all labeled differently by the $t$ integers in the set $T$.

The algorithm starts at the point $v$ with $T=\emptyset$. Let $l(v)$ be the label of $v$. When $l(v)=0, v$ is a discrete zero point by definition of the labeling rule and we are done. When $l(v) \neq 0$, the 0 -dimensional simplex $\{v\}$ is a $T$-complete facet of exactly one 1-dimensional simplex $\sigma\left(x^{1}, x^{2}\right)$ in $A(T)$ with $T=\{l(v)\}$, where $x^{1}=v$ and $x^{2}=v+e(l(v))$. Now, the algorithm generates, for varying sets $T \in \mathcal{I}$, a sequence of adjacent $t$-simplices in $A(T)$ with $T$-complete common facets. When the algorithm encounters a $t$-simplex $\sigma$ in $A(T)$ having a vertex carrying label $k$ and neither $k$ nor $-k$ belongs to $T$, then $k$ is added to $T$ and the algorithm continues in $A(T \cup\{k\})$ with the unique $(t+1)$-simplex in $A(T \cup\{k\})$ having $\sigma$ as its facet, while when the algorithm generates a $T$-complete facet $\tau$ lying on the boundary of $A(T)$ and therefore in $A(T \backslash\{k\})$ for some $k \in T$, then $k$ is deleted from $T$ and the algorithm continues in $A(T \backslash\{k\})$ with the facet opposite the vertex of $\tau$ carrying 
label $k$. Since each step is uniquely determined, the well-known Lemke-Howson argument implies that the algorithm will never visit a simplex more than once, see van der Laan and Talman (1981) for a more detailed description. Therefore, three possibilities may occur, namely (i) the algorithm generates a sequence of simplices going to infinity, or (ii) the algorithm generates a vertex carrying label $-k$ for some $k \in T$, i.e., it generates a simplex having a vertex with label $k$ and a vertex with label $-k$, for some $k \in N$, or (iii) it finds a vertex with label 0 . Clearly, in the third case the algorithm finds a discrete zero point of $f$. In the following we show that direction preserving and the negativity boundary condition prevent the algorithm from crossing the boundary of the set $C^{n}=\left\{x \in \mathbb{R}^{n} \mid m \leq x \leq M\right\}$ and therefore guarantees that the sequence of simplices generated by the algorithm will stay within $C^{n}$. Since the number of simplices in $C^{n}$ is finite and the algorithm will never visit a simplex more than once, within a finite number of steps the second or third case must occur. We then show that the second case is prevented by the direction preserving condition, implying that the algorithm must find a vertex with label 0 and thus a discrete zero point within a finite number of steps.

Lemma 3.3 Let $f: \mathbb{Z}^{n} \rightarrow \mathbb{R}^{n}$ be a direction preserving function satisfying the negativity boundary condition. Then no vertex of a T-complete facet of a simplex in $A(T)$ lies on the boundary of $C^{n}$

Proof: Let $\tau$ be a $T$-complete facet in $A(T)$ for some $T \in \mathcal{I}$ and take any vertex $x$ of $\tau$. By feature of the algorithm it holds that $x_{k} \leq v_{k}$ if $k$ is not in $T$ and that $x_{k} \geq v_{k}$ if $-k$ is not in $T$. So, if $x_{k}>v_{k}$, then we must have that $k \in T$ and hence there exists a vertex $y$ of $\tau$ with label $k$, implying that $f_{k}(y)>0$. Since $x$ and $y$ are cell connected, the direction preserving property implies that $f_{k}(x) \geq 0$. Similarly, if $x_{k}<v_{k}$, we must have $-k \in T$ and hence there exists a vertex $y$ of $\tau$ with label $-k$. So, $f_{k}(y)<0$ and thus $f_{k}(x) \leq 0$. Finally, if $x_{k}=v_{k}$, then we have $\left(x_{k}-v_{k}\right) f_{k}(x)=0$.

In conclusion, for any vertex $x$ of a $T$-complete facet $\tau$ in $A(T)$ we have that

$$
\begin{aligned}
(x-v) \cdot f(x) & =\sum_{h \in N}\left(x_{h}-v_{h}\right) f_{h}(x)=\sum_{\left\{h \mid x_{h}>v_{h}\right\}}\left(x_{h}-v_{h}\right) f_{h}(x) \\
& +\sum_{\left\{h \mid x_{h}<v_{h}\right\}}\left(x_{h}-v_{h}\right) f_{h}(x)+\sum_{\left\{h \mid x_{h}=v_{h}\right\}}\left(x_{h}-v_{h}\right) f_{h}(x) \geq 0,
\end{aligned}
$$

implying that $x$ can not be in $\operatorname{Bd}\left(C^{n}\right)$ because of the negativity boundary condition.

The lemma guarantees that the algorithm can not generate simplices outside the set $C^{n}$. Indeed, suppose that at some moment the algorithm generates a simplex outside $C^{n}$. Since the algorithm generates a sequence of adjacent simplices starting in the interior of $C^{n}$ and any two vertices of a simplex being generated are cell connected, this means that 
the algorithm must have generated before a simplex having at least one vertex in $\operatorname{Bd}\left(C^{n}\right)$, which is not possible according to the lemma.

Lemma 3.4 Let $f: \mathbb{Z}^{n} \rightarrow \mathbb{R}^{n}$ be a direction preserving function. Then there exists no simplex in $K^{\prime}(v)$ carrying labels $k$ and $-k$ for some $k \in N$.

Proof: Suppose to the contrary that there is a simplex carrying labels $k$ and $-k$ for some $k \in N$. Then for the vertex $x$ with label $k$ we have $f_{k}(x)>0$, while for the vertex $y$ with label $-k$ we have $f_{k}(y)<0$. Since $x$ and $y$ are cell connected, this contradicts the direction preserving property.

As a consequence of the two lemmas above, we obtain the following result, yielding a constructive proof for Theorem 3.1.

Proposition 3.5 Let $f: \mathbb{Z}^{n} \rightarrow \mathbb{R}^{n}$ be a direction preserving function satisfying the negativity boundary condition. Starting at the point $v$, the $2 n$-ray algorithm finds a discrete zero point of $f$ within a finite number of steps.

\section{Existence of discrete zero point under the antipodal boundary condition}

In this section we modify the 2 n-ray algorithm to provide a constructive and combinatorial proof for the following theorem given by Yang (2004b) for the more general class of locally gross direction preserving functions. The theorem can be seen as a discrete analogue of the celebrated Borsuk-Ulam theorem; for a similar version in the continuous setting, see for example van der Laan (1984) and Yang (1999).

Theorem 4.1 Let $f: \mathbb{Z}^{n} \rightarrow \mathbb{R}^{n}$ be a direction preserving function satisfying the antipodal boundary condition for some $u \in \mathbb{Z}^{n}$. Then $f$ has a discrete zero point $x^{*} \in \mathbb{Z}^{n}$, i.e., $f\left(x^{*}\right)=0^{n}$.

To apply a modified version of the 2 n-ray algorithm, we first extend the set $U^{n}$ to the set

$$
V^{n}=\left\{x \in \mathbb{R}^{n} \mid-\left(u_{i}+1\right) \leq x_{i} \leq u_{i}+1, \quad \forall i \in N\right\}
$$

and define the projection function $p: V^{n} \rightarrow U^{n}$ by

$$
p_{h}(x)=\max \left\{-u_{h}, \min \left\{u_{h}, x_{h}\right\}\right\}, \text { for all } h \in N .
$$

Clearly, $p(x)=x$ if $x \in U^{n}$. Moreover, $p(x) \in U^{n} \cap \mathbb{Z}^{n}$ if $x \in V^{n} \cap \mathbb{Z}^{n}$. We now define the function $g: V^{n} \cap \mathbb{Z}^{n} \rightarrow \mathbb{R}^{n}$ by setting $g(x)=f(x)$ for $x \in U^{n}$ and $g(x)=f(p(x))-f(-p(x))$ for $x \in V^{n} \backslash U^{n}$. Clearly, we have the following lemma. 
Lemma 4.2 The function $g$ satisfies $g(x)=-g(-x)$ for any $x \in \mathbb{Z}^{n} \cap \operatorname{Bd}\left(V^{n}\right)$.

Proof: Take any $x \in \mathbb{Z}^{n} \cap \operatorname{Bd}\left(V^{n}\right)$. Notice that $p(-x)=-p(x)$. Then we have

$$
\begin{aligned}
g(x) & =f(p(x))-f(-p(x))=-[f(-p(x))-f(p(x)] \\
& =-[f(p(-x))-f(-p(-x))]=-g(-x) .
\end{aligned}
$$

The lemma shows that $g$ satisfies the so-called antipodal property on the boundary of $V^{n}$, i.e., for a point $x$ on the boundary of $V^{n}$ the function value $g(x)$ is opposite to the function value $g(-x)$ of the antipodal point $-x$.

We now state the following lemma, saying that $g$ satisfies both the direction preserving and the antipodal boundary condition on $V^{n}$.

Lemma 4.3 Let $f: \mathbb{Z}^{n} \rightarrow \mathbb{R}^{n}$ be a direction preserving function satisfying the antipodal boundary condition for some $u \in \mathbb{Z}^{n}$. Then

(i) $g$ is direction preserving on $V^{n}$; and

(ii) $g$ satisfies the antipodal boundary condition on $V^{n}$.

Proof: Clearly, $g$ is direction preserving for any two cell connected points in $U^{n}$. It remains to consider the following two cases.

First, let $x, y \in \mathbb{Z}^{n}$ be two cell connected points on the boundary of $V^{n}$. Then we have to show both (i) and (ii). To do so, observe that each pair of the four points $x, y, p(x)$ and $p(y)$ are cell connected (it may happen that $p(x)=p(y)$ ). For given $h \in N$, first suppose that not both $f_{h}(p(x))$ and $f_{h}(p(y))$ are equal to zero. W.l.o.g. we may assume that $f_{h}(p(x)) \neq 0$. If $f_{h}(p(x))>0$, then $f_{h}(p(y)) \geq 0$ since $f$ is direction preserving on $U^{n}$, while the antipodal boundary condition of $f$ on $U^{n}$ implies that $f_{h}(-p(x)) \leq 0$ and $f_{h}(-p(y)) \leq 0$. Thus, $g_{h}(x)=f_{h}(p(x))-f_{h}(-p(x)) \geq 0$ and similarly $g_{h}(y) \geq 0$. Hence $g_{h}(x) g_{h}(y) \geq 0$, showing that $g$ is direction preserving for the two cell connected points $x$ and $y$ on boundary $V^{n}$. Also, by Lemma $4.2, g_{h}(x) g_{h}(-y)=-g_{h}(x) g_{h}(y) \leq 0$, showing that also the antipodal boundary condition is satisfied. Similarly, it follows that $g$ satisfies (i) and (ii) for $x$ and $y$ when $f_{h}(p(x))<0$. It remains to consider the case that both $f_{h}(p(x))=0$ and $f_{h}(p(y))=0$. Since also $-p(x)$ and $-p(y)$ are cell connected and $f$ is direction perserving, it then follows that $g_{h}(x) g_{h}(y)=f_{h}(-p(x)) f_{h}(-p(y)) \geq 0$ and thus $g$ is direction preserving for $x$ and $y$. Also, by Lemma 4.2, $g_{h}(x) g_{h}(-y)=-g_{h}(x) g_{h}(y) \leq 0$, showing that the antipodal boundary condition is satisfied.

Second, let $x, y \in \mathbb{Z}^{n}$ be two cell connected points with $x$ on the boundary of $U^{n}$ and $y$ on the boundary of $V^{n}$. Now, $x$ is in the interior of $V^{n}$, so we only have to show that $g$ satisfies the direction preserving property for $x$ and $y$. Clearly, any pair of two of the 
points $x, y$ and $p(y)$ are cell connected. In case $f_{h}(x)>0$, we have $f_{h}(p(y)) \geq 0$ since $f$ is direction preserving. Further, $f_{h}(-p(y)) \leq 0$ since $f$ satisfies the antipodal boundary condition. Then, $g_{h}(y)=f_{h}(p(y))-f_{h}(-p(y)) \geq 0$ and thus $g_{h}(x) g_{h}(y)=f_{h}(x) g_{h}(y) \geq 0$, showing that $g$ is direction preserving for $x$ and $y$. Similarly the result follows when $f_{h}(x)<0$. If $f_{h}(x)=0$, we have $g_{h}(x)=f_{h}(x)=0$ and thus $g_{h}(x) g_{h}(y)=0$.

We now apply the algorithm as given in the previous section on $K^{\prime}(w)$ with $w=0^{n}$. So, for any $T \in \mathcal{I}$, the set $A(T)$ is given by

$$
\begin{gathered}
A(T)=\left\{x \in V^{n} \mid \quad x_{i} \geq 0 \text { if } i \in T ; \quad x_{i} \leq 0 \text { if }-i \in T ;\right. \\
\text { and } \left.x_{i}=0 \text { otherwise }\right\} .
\end{gathered}
$$

We have to make one modification, called the reflection step. Observe that if $\tau$ is a $T$ complete facet of a $t$-simplex in $A(T)$ and lies on the boundary of $V^{n}$, then the antipodal facet $-\tau$ also lies on the boundary of $V^{n}$ and is a $(-T)$-complete facet of precisely one $t$-simplex in $A(-T)$. Clearly, the latter fact follows immediately from Lemma 4.2 and the labeling rule for the function $g$. Therefore, if the algorithm hits the boundary of $V^{n}$ with a $T$-complete facet $\tau$ in $A(T)$, it continues with the $(-T)$-complete $-\tau$, being a facet of some unique $t$-simplex in $A(-T)$. This reflection step is crucial in the algorithm and has been previously used by for example van der Laan (1984) to find an approximate zero point of a continuous function on $U^{n}$.

Similar as in the previous section, the algorithm starts at the origin $0^{n}$ with $T=\emptyset$. Let $l\left(0^{n}\right)$ be the label of $0^{n}$. When $l\left(0^{n}\right)=0$, then $0^{n}$ is a discrete zero point. Otherwise, $\left\{0^{n}\right\}$ is a $T$-complete facet of precisely one 1-dimensional simplex $\sigma\left(x^{1}, x^{2}\right)$ in $A(T)$ with $T=\left\{l\left(0^{n}\right)\right\}$, where $x^{1}=0^{n}$ and $x^{2}=e(l(v))$. Again, the algorithm generates, for varying sets $T \in \mathcal{I}$, a sequence of adjacent $t$-simplices with $T$-complete common facets in $A(T)$, making a reflection step every time the algorithm generates a $T$-complete facet on the boundary of $V^{n}$. Again each step is uniquely determined and the well-known LemkeHowson argument implies that the algorithm will never visit a simplex more than once; see van der Laan (1984) for a more detailed discussion. Since the algorithm stays within $V^{n}$ and the number of simplices within $V^{n}$ is finite, only two possibilities may occur, namely, (i) the algorithm generates a vertex having label $-k$ for some $k \in T$; or (ii) it finds a vertex with label 0 . However, since $g$ is direction preserving on $V^{n}$, the first case can not occur and thus the algorithm must stop with an integral point $x$ in $V^{n}$ with label 0 . The next lemma shows that then $p(x) \in U^{n}$ is a discrete zero point of $f$.

Lemma 4.4 Let $x \in \mathbb{Z}^{n} \cap V^{n}$ be a vertex of the $K^{\prime}\left(0^{n}\right)$-triangulation with label 0 . Then $p(x)$ is a discrete zero point of $f$.

Proof: Notice that $p(x)$ is an integral vector of $U^{n}$. Since $l(x)=0$ we have that $g(x)=0^{n}$. If $x \in U^{n}$, then $g(x)=f(x)$ and $p(x)=x$ imply that $f(p(x))=f(x)=0^{n}$. Otherwise, 
$x \in \operatorname{Bd}\left(V^{n}\right)$. Then $g(x)=f(p(x))-f(-p(x))=0^{n}$, i.e., $f(p(x))=f(-p(x))$. Because $f_{h}(p(x)) f_{h}(-p(x)) \leq 0$ for every $h \in N$, we must have $f(p(x))=f(-p(x))=0^{n}$.

Observe that if $l(x)=0$ and $x \in \operatorname{Bd}\left(V^{n}\right)$, then both $p(x)$ and $-p(x)$ are discrete zero points of $f$ on the boundary of $U^{n}$.

As a consequence of the lemma above, we obtain the following result, yielding a constructive proof for Theorem 4.1 .

Proposition 4.5 Let $f: \mathbb{Z}^{n} \rightarrow \mathbb{R}^{n}$ be a direction preserving function satisfying the antipodal boundary condition on $U^{n}$. Starting from the origin $0^{n}$, the $2 n$-ray algorithm with reflection step finds a discrete zero point of $f$ within a finite number of steps.

\section{Concluding remarks}

In this paper we have presented an algorithm to find a discrete zero point of a function $f: \mathbb{Z}^{n} \rightarrow \mathbb{R}^{n}$ under two different boundary conditions. The algorithm starts with a given integral point in $\mathbb{Z}^{n}$ and terminates with an exact discrete zero point of the function within a finite number of steps. It is worth pointing out that the current study and those of Iimura (2003) and Yang (2004a, b) were motivated by and closely related to the rich literature on economies with indivisibilities; see, e.g., Scarf (1981), Kelso and Crawford (1982), van der Laan, Talman and Yang (2002) among others. As a byproduct, the current paper provides an efficient method for finding a discrete equilibrium in the spatial price equilibrium model studied in Yang (2004b) and related models as well.

The algorithm we used in this paper is a so-called integer labeling 2n-ray simplicial algorithm. Two other simplicial algorithms which can also use integer labeling rule are the $(n+1)$-ray algorithm of van der Laan and Talman $(1979,1981)$ and the 2-ray algorithm of Yamamoto (1983). However, it is not clear if these methods can also find a discrete zero point of a function under similar conditions we have studied here. To deal with the more general class of locally gross direction preserving functions, we need the more sophisticated concept of so-called vector labeling algorithms. In a subsequent paper we will provide such an algorithm, and thus constructive existence proofs, for the class of locally gross direction preserving functions. 


\section{References}

[1] Allgower, E.L., Georg, K., 1990. Numerical Continuation Methods: An Introduction, Springer, Berlin.

[2] Danilov, V., Koshevoy, G., 2004. Existence theorem of zero point in a discrete case, Moscow, draft.

[3] Eaves, B.C., 1972. Homotopies for computation of fixed points, Mathematical Programming 3, 1-22.

[4] Iimura, T., 2003. A discrete fixed point theorem and its applications, Journal of Mathematical Economics 39, 725-742.

[5] Iimura, T., Murota, K., Tamura, A., 2004. Discrete fixed point theorem reconsidered, METR 2004-09, University of Tokyo, Tokyo.

[6] Kelso, A., Crawford, V., 1982. Job matching coalition formation and gross substitutes, Econometrica 50, 1483-1504.

[7] Laan van der, G., 1984. On the existence and approximation of zeros, Mathematical Programming 28, 1-14.

[8] Laan van der, G., Talman, A.J.J., 1979. A restart algorithm for computing fixed points without an extra dimension, Mathematical Programming 17, 74-84.

[9] Laan van der, G., Talman, A.J.J., 1981. A class of simplicial restart fixed point algorithms without an extra dimension, Mathematical Programming 20, 33-48.

[10] Laan van der, G., Talman, A.J.J., Yang, Z., 2002. Existence and welfare properties of equilibrium in an exchange economy with multiple divisible and indivisible commodities and linear production technologies, Journal of Economic Theory 103, 411-428.

[11] Merrill, O.H., 1972. Applications and Extensions of an Algorithm that Computes Fixed Points of Certain Upper Semi-Continuous Point-to-Set Mappings, PhD Thesis, University of Michigan, Ann Arbor.

[12] Scarf, H., 1967. The approximation of fixed points of a continuous mapping, SIAM Journal on Applied Mathematics 15, 1328-1343.

[13] Scarf, H., 1973. The Computation of Economic Equilibria, Yale University Press, New Haven. 
[14] Scarf, H., 1981. Production sets with indivisibilities-part I: generalities, Econometrica 49, 1-32.

[15] Todd, M.J., 1976. Computation of Fixed Points and Applications, Springer-Verlag, Berlin.

[16] Todd, M.J., 1978. Improving the convergence of fixed point algorithms, Mathematical Programming Study 7, 151-179.

[17] Yamamoto, Y., 1983. A new variable dimension algorithm for the fixed point problem, Mathematical Programming 25, 329-342.

[18] Yang, Z., 1999. Computing Equilibria and Fixed Points, Kluwer, Boston.

[19] Yang, Z., 2004a. Discrete nonlinear complementarity problems, FBA Working Paper No. 205, Yokohama National University, Yokohama.

[20] Yang, Z., 2004b. Discrete fixed point analysis and its applications, FBA Working Paper No. 210, Yokohama National University, Yokohama. 